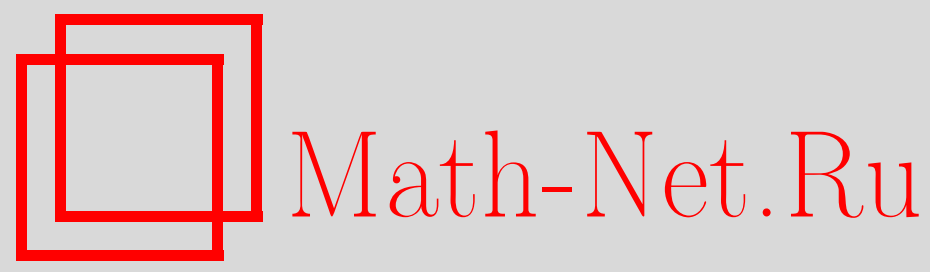

С. В. Колесников, Некоторые замечания о дескриптивнометрических характеристиках особых множеств аналитических функций, Матем. заметки, 1998, том 63, выпуск 1, 56-61

DOI: https://doi.org/10.4213/mzm1247

Использование Общероссийского математического портала Math-Net.Ru подразумевает, что вы прочитали и согласны с пользовательским соглашением http://www . mathnet.ru/rus/agreement

Параметры загрузки:

IP : 3.95 .254 .165

26 апреля 2023 г., 16:14:03

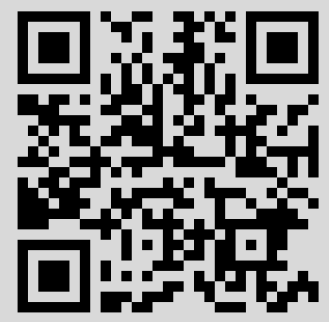




\section{НЕКОТОРЫЕ ЗАМЕЧАНИЯ \\ О ДЕСКРИПТИВНО-МЕТРИЧЕСКИХ ХАРАКТЕРИСТИКАХ ОСОБЫХ МНОЖЕСТВ АНАЛИТИЧЕСКИХ ФУНКЦИЙ}

\section{С. В. Колесников}

Настоящая работа содержит два замечания о строении особых граничных множеств функций, аналитических в единичном круге $D:|z|<1$. Первое замечание касается вопроса об обращении теоремы Плеснера. Доказано, что для того чтобы три попарно не пересекающихся множества $E_{1}, E_{2}$ и $E_{3}, \bigcup_{i=1}^{3} E_{i}=\Gamma$, единичной окружности $\Gamma$ : $|z|=1$, были соответственно множествами всех точек Плеснера $-I(f)$, всех точек $\Phi$ ату $-F(f)$ и множеством всех исключительных граничных точек $-E(f)$ для некоторой голоморфной в $D$ функции $f$, необходимо и достаточно, чтобы $E_{1}$ имело тип $G_{\delta}$, a $E_{3}$ имело тип $G_{\delta \sigma}$ и линейную меру нуль. Во второй части работы показьвается, что для любого подмножества $E$ единичной окружности $\Gamma$, имеющего нулевую логарифмическую емкость и тип $G_{\delta \sigma}$ на $\Gamma$, существует однолистная в $D$ функция, у которой угловые пределы не существуют в точках множества $E$ и существуют в остальных точках окружности $\Gamma$.

Библиограффия: 10 названий.

Настоящая работа содержит два замечания о строении множеств граничных особых точек функций, аналитических в единичном круге $D:|z|<1$. Первое замечание касается обращения теоремы Плеснера об особых граничных точках функций, мероморфных в $D$. Во второй части работыпоказьвается, что для любого подмножества $E$ единичной окружности $\Gamma$, типа $G_{\delta}$ и нулевой логарифмической емкости, существует однолистная в $D$ функция, не имеющая угловых пределов на $E$ и имеющая их в остальных точках окружности $Г$.

1. Пусть $f(z)$ - функция, определенная в круге $D ; \Gamma$ - единичная окружность $|z|=1$; $\zeta \in \Gamma$. Радиальным пределом функции $f(z)$ в $\zeta$ назьвается предел

$$
\lim _{r \rightarrow 1-0} f(r \zeta) \text {. }
$$

Говорят, что функция $f(z)$ имеет в точке $\zeta$ угловой предел, если $f(z)$ имеет предел при $z \rightarrow \zeta$ по любому углу с вершиной в $\zeta$, образованному хордами круга $D$.

В 1906 году П. Фату [1] доказал, что если $f(z)$ ограничена и аналитична в круге $D$, то она почти всюду на окружности Г имеет радиальные пределы, а следовательно, по теореме Линделефа $f(z)$ имеет почти всюду и угловые пределы. Н.Н. Лузин в [2] показал, что этот результат Фату является точным: для любого множества $E \subset \Gamma$ существует ограниченная аналитическая функция, не имеющая угловых пределов в точках множества $E$.

Точки $\zeta \in \Gamma$, в которых функция $f(z)$ имеет угловые пределы, называются точками $\Phi a m y$. Множество всех точек Фату функции $f(z)$ будем обозначать через $F(f)$. Точками Плеснера функции $f$ называются такие точки $\zeta$, в которых предельное множество 
по любому углу с вершиной в $\zeta$ совпадает с расширенной комплексной плоскостью $\overline{\mathbb{C}}$. Множество всех точек Плеснера функции $f$ обозначим через $I(f)$.

И.И. Плеснером [3] было показано, что для любой мероморфной в $D$ функции $f$ почти каждая точка окружности Г является или точкой Фату, или точкой Плеснера. Таким образом, окружность Г представляется в виде объединения множества Плеснера $I(f)$, множества Фату $F(f)$ и некоторого исключительного множества $E(f)$, имеющего линейную лебегову меру нуль. Заметим, что если $f(z)$ ограничена в $D$, то $E(f)$ является множеством всех точек, в которых $f$ не имеет угловых пределов.

Известно, что множество $F(f)$ имеет тип $F_{\sigma \delta}$ на окружности Г. В 1970 году П. Лаппаном [4] было доказано, что для мероморфных функций множество Плеснера имеет тип $G_{\delta}$ и что для любого множества $E$ типа $G_{\delta}$ существует голоморфная функция $f(z)$, для которой $E=I(f)$. Отсюда, в частности, следует, что множество $E(f)$ имеет тип $G_{\delta \sigma}$. В примере Лаппана точки множества $\Gamma \backslash I(f)$, вообще говоря, могут быть как точками Фату, так и точками из $E(f)$.

Рядом авторов рассматривался вопрос о совместной характеристике всех трех множеств. В частности, А. Н. Канатниковым [5] было показано, что для любых трех попарно непересекающихся множеств $E_{1}, E_{2}$ и $E_{3}, \bigcup_{i=1}^{3} E_{i}=\Gamma$, таких, что $E_{1}$ имеет тип $G_{\delta}$, а $E_{3}$ - тип $F_{\sigma}$ и линейную меру нуль, существует мероморфная в круге $D$ функция $f$ такая, что $E_{1}=I(f), E_{2}=F(f)$ и $E_{3}=E(f)$. При этом для доказательства им применялся следуюший результат Ловатера и Пираняна: для любого множества $E \subset \Gamma$, имеющего нулевую линейную меру и тип $F_{\sigma}$, существует аналитическая и ограниченная в круге $D$ функция $f(z)$ такая, что $E=E(f)[6]$.

Используя полученную автором (см. [7]) полную характеристику множеств $E(f)$ для функций $f$, аналитических и ограниченных в круге, методом, аналогичным используемому в [5], легко дать полную совместную характеристику множеств $I(f), F(f)$ и $E(f)$ для голоморфных в $D$ функций. Как следствие, отсюда получается полная характеристика множеств всех точек, в которых голоморфные в круге $D$ функции не имеют конечных угловых пределов.

Теорема 1. Для того чтобъ три попарно не пересекающихся множсества $E_{1}$, $E_{2}$ и $E_{3}, \bigcup_{i=1}^{3} E_{i}=\Gamma$, били соответственно мнохествами $I(f), F(f)$ и $E(f)$ для некоторой голоморфной в $D$ функиии $f$, необходимо и достаточно, чтобы $E_{1}$ имело тип $G_{\delta}$, а $E_{3}$ имело тип $G_{\delta \sigma}$ и линейную меру нуль.

ДокАЗАТЕЛЬСтво. В [7] при доказательстве теоремы 3 показьвается, что любое множество типа $G_{\delta}$ на $\Gamma$ является множеством Плеснера некоторой голоморфной в $D$ функции, причем функцию можно выбрать так, что все точки, не принадлежащие этому множеству, будут точками Фату.

Пусть $f_{1}$ - такая функция для множества $E_{1}$.

По теореме 1 из [8] для множества $E_{3}$ найдется ограниченная голоморфная в $D$ функция $f_{3}$, не имеющая на $E_{3}$ угловых пределов и имеющая их в каждой точке множества $\Gamma \backslash E_{3}$. Положим $f(z)=f_{1}(z)+f_{3}(z)$.

В точках $\zeta \in E_{1}$ по любому углу $V$ с вершиной $\zeta$, образованному хордами круга $D$, предельное множество функции $f_{1}$ есть расширенная комплексная плоскость $\overline{\mathbb{C}}$, а предельное множество функции $f_{3}$ состоит из одной точки. Очевидно, предельное множество функции $f$ в точке $\zeta$ по углу $V$ есть $\overline{\mathbb{C}}$. Таким образом, все точки $\zeta \in E_{1}$ являются точками Плеснера. Если $\zeta \in E_{3}$, то функция $f_{1}$ имеет в $\zeta$ конечный предел по углу $V$, a $f_{3}$ не имеет предела. Поэтому функция $f$ также не имеет угловьх пределов в точках $\zeta \in E_{3}$. Поскольку $f_{3}$ ограничена, то угловое предельное множество функции $f$ не может быть плоскостью $\overline{\mathbb{C}}$. Таким образом, $E_{3}$ состоит из исключительных особых точек 
функции $f$.

Наконец, все точки множества $E_{2}$ являются точками Фату функции $f$, поскольку таковьми они являются для обеих функций $f_{1}$ и $f_{3}$.

Теорема доказана.

СлЕДСТВИЕ. Для того чтобы множество $Е \subset$ Г являлось множсеством всех точек, в которых нет конечных угловых пределов у некоторой голоморфной в $D$ функиии, необходимо и достаточно, чтобы оно было представимо в виде обвединения двух множеств - мнохества типа $G_{\delta}$ и множсества типа $G_{\delta \sigma}$ линейной мерь нуль.

2. Пусть $E$ - некоторое ограниченное борелевское множество на комплексной плоскости; $\mu$ - положительная борелевская мера на $E$. Логарифмическим потенииалом $U_{\mu}(z)$ будем назьвать интеграл

$$
U_{\mu}(z)=\int_{E} \ln \frac{1}{|\zeta-z|} d \mu(\zeta) .
$$

Логарифмической емкостью множества $E$ называется величина

$$
\operatorname{Cap} E=\sup \mu(E) \text {, }
$$

где супремум берется по всем положительньм борелевским мерам, сосредоточенным на $E$, для которых $U_{\mu}(z) \leqslant 1$.

А. Берлингом [9] было доказано, что если функция $f(z)$ однолистна в круге $D$, то она имеет конечные угловые пределы во всех точках окружности Г за исключением множества точек логарифмической емкости нуль. Таким образом, для однолистной в $D$ функции $f$ множество $E(f)$ имеет тип $G_{\delta \sigma}$ и нулевую логарифмическую емкость.

Ниже дается достаточное условие на множество для того, чтобы оно было множеством $E(f)$ для некоторой функции, однолистной в $D$.

Теорема 2. Для любого множества $E \subset \Gamma$, имеющего нулевую логарифмическую емкость и тип $G_{\delta}$ на окружности $\Gamma$, существует ограниченная однолистная в круге $D$ функиия $f(z)$ такая, что $E=E(f)$.

ДокАЗАТЕЛЬСТво. Покажем сначала, что для любого $\varepsilon>0$ и любого открытого на $\Gamma$ множества $O \supset E$ существуют открытое множество $\widetilde{O}$ и положительная борелевская мера $\mu, \mu(\Gamma)<\varepsilon$, такие, что $E \subset \widetilde{O} \subset O$, а потенщиал $U_{\mu}(z)$ ограничен и удовлетворяет условиям:

$$
U_{\mu}(z) \geqslant 1, \quad z \in \widetilde{O} ; \quad U_{\mu}(z)<\varepsilon, \quad z \in \Gamma \backslash O .
$$

Для доказательства каждую из открытых дуг, составляющих множество $O$, двумя последовательностями, сходящимися к ее концам, разобьем на открытые дуги. При этом, так как Cap $E=0$, а логарифмическая емкость любой невырожденной дуги положительна, то точки разбиения можно выбрать из $\Gamma \backslash E$. Совокупность всех полученных дуг расположим в последовательность $\gamma_{n}, n=1,2, \ldots$ Обозначим через $\rho_{n}$ расстояние от дуги $\gamma_{n}$ до ближайшей дуги, не смежной с $\gamma_{n}$.

Поскольку логарифмическая емкость пересечения $E \cap \gamma_{n}$ равна 0 и борелевские множества измеримы относительно емкости, то можно найти открытое множество $\widetilde{O}_{n} \subset \gamma_{n}$ такое, что $E \cap \gamma_{n} \subset \widetilde{O}_{n}$ и

$$
\text { Cap } \widetilde{O}_{n}<\frac{\varepsilon}{M 2^{n}}, \quad \text { где } M_{n}=\max \left\{1, \ln \frac{1}{\rho_{n}}\right\} \text {. }
$$


Пусть $\widetilde{\mu}_{n}-$ равновесная мера для $\widetilde{O}_{n}, n=1,2, \ldots, \mu=\sum_{n=1}^{\infty} \widetilde{\mu}_{n}$. Так как $\widetilde{\mu}_{n}-$ равновесная мера, то $\widetilde{\mu}_{n}(\Gamma)=\operatorname{Cap} \widetilde{\mu}_{n}(\Gamma)$, и согласно $(1)$

$$
\mu(\Gamma)=\sum_{n=1}^{\infty} \widetilde{\mu}_{n}(\Gamma)<\sum_{n=1}^{\infty} \frac{\varepsilon}{M_{n} 2^{n}} \leqslant \varepsilon .
$$

Рассмотрим потенциал $U_{\mu}(z)$. Так как $U_{\widetilde{\mu}_{n}}-$ равновесньй потенциал для $\widetilde{O}_{n}$, то $U_{\widetilde{\mu}_{n}}(z)=1$ во внутренних точках множества $\widetilde{O}_{n}$. Отсюда следует, что на множестве $\widetilde{O}=\bigcup_{n=1}^{\infty} \widetilde{O}_{n}$ имеет место неравенство

$$
U_{\mu}(z)=\sum_{n=1}^{\infty} U_{\widetilde{\mu}_{n}}(z) \geqslant 1
$$

Из неравенства (1) следует, что для всех точек $z \in \Gamma$, находящихся от $\gamma_{n}$ на расстоянии $>\rho_{n}$, вьполнено

$$
U_{\widetilde{\mu}_{n}}(z)=\int_{\Gamma} \ln \frac{1}{|\zeta-z|} d \widetilde{\mu}_{n}(\zeta)<\int_{\Gamma} \ln \frac{1}{\rho_{n}} d \widetilde{\mu}_{n}=M_{n} \widetilde{\mu}_{n}\left(\widetilde{O}_{n}\right)=M_{n} \operatorname{Cap} \widetilde{O}_{n}<\frac{\varepsilon}{2^{n}} .
$$

Поскольку точки $z \in \Gamma \backslash O$ находятся от дуги $\gamma_{n}$ на расстоянии, большем $\rho_{n}, n=1,2, \ldots$, из (2) следует, что на $\Gamma \backslash O$ имеет место неравенство

$$
U_{\mu}(z)=\sum_{n=1}^{\infty} U_{\widetilde{\mu}_{n}}(z) \leqslant \sum_{n=1}^{\infty} \frac{\varepsilon}{2^{n}}=\varepsilon .
$$

Наконец, если $z \in O \backslash \widetilde{O}$, то (2) может не вьполняться только для индексов $n$, соответствующих дуге $\gamma_{n}$, содержащей $z$, или дугам, смежным с ней, т.е. лишь для трех индексов. Отсюда получается, что потенциал $U_{\mu}(z)$ ограничен: $U_{\mu}(z)<3+\varepsilon$, и требуемое утверждение доказано.

Поскольку множество $E$ имеет тип $G_{\delta}$, то, применяя доказанное утверждение, нетрудно построить последовательность открытых на $\Gamma$ множеств $O_{1} \supset O_{2} \supset \ldots \supset$ $O_{n} \supset \cdots$, пересечение которых равно $E\left(E=\bigcap_{n=1}^{\infty} O_{n}\right)$, и меры $\mu_{n}, \mu_{n}(\Gamma)<1 / 2^{n}$, так, что потенциал $U_{\mu_{n}}$ ограничен, удовлетворяет неравенству $U_{\mu_{n}}(z) \geqslant 1$ на множестве $O_{n}$ и неравенству $U_{\mu_{n}}(z)<1 / 2^{n}$ на $\Gamma \backslash O_{n-1}, n=1,2, \ldots$

Положим $\mu=\sum_{n=1}^{\infty} \mu_{n}$. Тогда

$$
U_{\mu}(z)=\sum_{n=1}^{\infty} U_{\mu_{n}}(z)
$$

Если $z \in E$, то по построению мер $\mu_{n} U_{\mu_{n}}(z)>1$ и потенциал $U_{\mu}(z)$ равен $+\infty$. Отсюда в силу непрерьвности снизу потенциала $U_{\mu}$ следует, что $U_{\mu}(z)$ имеет в каждой точке множества $E$ предел, равньй $+\infty$.

Если $z \in \Gamma \backslash E$, то существует номер $N$ такой, что $z \notin O_{n}$ при $n>N$. Тогда на $\Gamma \backslash O_{N}$ выполнено $U_{\mu_{n}}(z)<1 / 2^{n}, n>N$, и

$$
U_{\mu}(z)=\sum_{n=1}^{N} U_{\mu_{n}}(z)+\sum_{N+1}^{\infty} U_{\mu_{n}}(z)<\sum_{n=1}^{N} U_{\mu_{n}}(z)+1
$$


Поскольку все потенциалы $U_{\mu_{n}}$ ограничены, то $U_{\mu}(z)<+\infty$, т.е. потенциал $U_{\mu}$ конечен в каждой точке множества $\Gamma \backslash E$.

Положим

$$
F(z)=\int_{\Gamma} \ln \frac{1}{\zeta-z} d \mu(\zeta),
$$

где ветвь логарифма выбирается так, что $0 \leqslant \operatorname{Im} \ln (1 / \zeta)<2 \pi$.

Очевидно, $F(z)$ голоморфна в круге $D, \operatorname{Re} F(z)=U_{\mu}(z)$ и $F(z)$ имеет бесконечные пределы в каждой точке множества $E$.

Покажем, что если $z_{0} \in \Gamma \backslash E$, то функция $F(z)$ имеет в точке $z_{0}$ конечный угловой предел.

Пусть $V$ - угол с вершиной в $z_{0}$ величины $2 \varphi$, симметричньй относительно радиуса круга $D$ с конщом в $z_{0} ; z \in V \cap D,\left|z-z_{0}\right|<\cos \varphi$. Нетрудно видеть, что при $\zeta \in \Gamma$ выполнено $\left|\zeta-z_{0}\right| /|\zeta-z|<1 / \cos \varphi$.

Отсюда по выбору ветви $\ln (1 /(\zeta-z))$ имеем

$$
\begin{aligned}
\left|\ln \frac{1}{\zeta-z}\right| & \leqslant\left|\operatorname{Re} \ln \frac{1}{\zeta-z}\right|+\left|\operatorname{Im} \ln \frac{1}{\zeta-z}\right| \leqslant\left|\ln \frac{1}{|\zeta-z|}\right|+4 \pi \leqslant \ln \frac{2}{|\zeta-z|}+4 \pi \\
& =\ln \frac{1}{|\zeta-z|}+\ln 2+4 \pi=\ln \frac{1}{\left|\zeta-z_{0}\right|}+\ln \frac{\left|\zeta-z_{0}\right|}{|\zeta-z|}+\ln 2+4 \pi \\
& \leqslant \ln \frac{1}{\left|\zeta-z_{0}\right|}+\ln \frac{1}{\cos \varphi}+\ln 2+4 \pi .
\end{aligned}
$$

Поскольку интеграл

$$
U_{\mu}\left(z_{0}\right)=\int_{\Gamma} \ln \frac{1}{\left|\zeta-z_{0}\right|} d \mu(\zeta)
$$

конечен, отсюда следует, что при всех $z \in V \cap D,\left|z-z_{0}\right|<\cos \varphi\left(\right.$ а также и при $\left.z=z_{0}\right)$, функция $|\ln (1 /(\zeta-z))|$ мажорируется функцией, суммируемой на Г по мере $\mu$.

Так как $1 /(\zeta-z) \rightarrow 1 /\left(\zeta-z_{0}\right)$ при $z \rightarrow z_{0}$, применяя теорему Лебега о предельном переходе под знаком интеграла, получим, что

$$
F(z) \rightarrow F\left(z_{0}\right)=\int_{\Gamma} \frac{1}{\zeta-z_{0}} d \mu(\zeta),
$$

когда $z \rightarrow z_{0}$, оставаясь внутри угла $V$.

Далее, нетрудно видеть, что

$$
\begin{aligned}
|F(z)| & \leqslant \int_{\Gamma}\left|\operatorname{Re} \ln \frac{1}{\zeta-z}\right| d \mu(\zeta)+\int_{\Gamma}\left|\operatorname{Im} \ln \frac{1}{\zeta-z}\right| d \mu(\zeta) \\
& \leqslant \int_{\Gamma}\left|\ln \frac{1}{|\zeta-z|}\right| d \mu(\zeta)+4 \pi \mu(\Gamma) \leqslant \mu(\Gamma) \ln \frac{1}{1-|z|}+4 \pi \mu(\Gamma) .
\end{aligned}
$$

Пусть

$$
q(z)=\int_{0}^{z} z F(z) d z
$$

Очевидно, из предыдущего неравенства следует, что $q(z)$ непрерывна в замкнутом круге $|z| \leqslant 1$.

Положим

$$
g(z)=z F(z)-q(z)+\frac{\mu(\Gamma)}{2} z
$$


Эта функция, как и $F(z)$, имеет на множестве $E$ бесконечные пределы, а на его дополнении $\Gamma \backslash E$ - конечные угловые пределы. Дифференцируя $g(z)$, получим

$$
\operatorname{Re} g^{\prime}(z)=\frac{1}{2} \int_{\Gamma} \frac{1-r^{2}}{1-2 r \cos (t-\theta)+r^{2}} d \mu(\zeta)>0
$$

где $\zeta=e^{i t}$, a $z=r e^{i \theta}$.

Отсюда по известному критерию однолистности (см., например, [10]) следует, что $g$ однолистна в круге $D$.

Пусть $G$ - область, на которую функция $g$ отображает единичный круг; $w_{0}$ - некоторая конечная граничная точка области $G$. Обозначим через $K$ связную компоненту множества всех конечных граничных точек области $G$, а через $G_{1}$ ту компоненту дополнения к $K$, которая содержит $G$. При этом бесконечная точка принадлежит носителю только одного простого конца области $G_{1}$. Пусть $\tau(z)$ - функция, отображающая $G_{1}$ конформно на круг с разрезом по гладкой спирали, разматывающейся к окружности $\Gamma$ так, что простой конец области $G_{1}$, соответствующий бесконечной граничной точке, переходит в простой конец круга с разрезом, соответствуюший окружности. Тогда функция $\tau(z)$ не будет иметь предела по любому пути, ведущему в бесконечную точку, и будет иметь предел по любому пути с концом в конечной точке границы области $G_{1}$. Отсюда, так как $g(z)$ имеет бесконечные пределы в каждой точке множества $E$ и конечные пределы в остальных точках окружности $\Gamma$, то функция $f(z)=\tau(g(z))$ не имеет радиальных пределов на $E$ и имеет их на $\Gamma \backslash E$. Таким образом, однолистная функция $f(z)$ является искомой.

Теорема доказана.

\section{СПИСОК ЦИТИРОВАННОЙ ЛИТЕРАТУРЫ}

[1] Fatou P. Series trigonometriques et series de Taylor // Acta Math. 1906. V. 30. P. 335-400.

[2] Лузин Н. H. Sur la représentation conforme // J. Reine Angew. Math. 1919. V. 158. P. 77-80.

[3] Plessner A. Über das Verhalten analytischer Functionen am Rande ihres Definitionsbereichs // J. Reine Angew. Math. 1927. V. 158. P. 219-227.

[4] Lappan P. A characterization of Plessner points // Bull. London Math. Soc. 1970. V. 2. P. $60-62$.

[5] Канатников А. Н. Обращение теоремы Мейера для мероморфных функций // Докл. АН CCCP. 1978. T. 238. № 5. C. 1043-1046.

[6] Lohwater A. J., Piranian G. The boundary behavior of functions analytic in a disk // Acad. Sci. Fenn. Ser. A I. Math. 1957. V. 239. P. 1-17.

[7] Хасан А. А.-Р., Канатников А. Н. Обращение теоремы Мейера для произвольных направлений // Вестн. МГУ. Сер. 1. Матем., мех. 1984. № 5. С. 38-41.

[8] Колесников С. В. О множествах несуществования радиальных пределов ограниченных аналитических функций // Матем. сб. 1994. Т. 185. № 4. С. 91-100.

[9] Beurling A. Ensembles exceptionnels // Acta Math. 1940. V. 72. P. 1-13.

[10] Фёдоров В. С. О производной комплексной функции // Докл. АН СССР. 1948. Т. 63. № 1. C. $34-36$. 